\title{
Hashimoto Thyroiditis: Simple Diagnosis with 2D-Shear Wave Elastography
}

\author{
Düzgün Yıldırım1, Deniz Esin Tekcan Şanlı2*, Deniz Aliş ${ }^{3}$, Mutlu Şahin', Alev Bakır5, \\ Ahmet Erdem Kılavuz 6
}

\author{
${ }^{1}$ Department of Medical Imaging, Vocational School of Health Sciences, Acıbadem University, Istanbul, Turkey \\ ${ }^{2}$ Department of Radiology, Acıbadem Kozyatağ \\ ${ }^{3}$ Department of Radiology, Istanbul Mehmet Akif Ersoy Thoracic and Cardiovascular Surgery Research and Training Hospital, \\ Istanbul, Turkey \\ ${ }^{4}$ Department of General Surgery, Dışkapı Yıldırım Beyazıt Education and Research Hospital, Ankara, Turkey \\ ${ }^{5}$ Biostatistics and Medical Informatics Department, Haliç University, Istanbul, Turkey \\ ${ }^{6}$ Department of Otorhinolaryngology, Acibadem University, Istanbul, Turkey \\ Email: yildirimduzgun@yahoo.com, ‘tekcandenizesin@gmail.com,drdenizalis@gmail.com,drmutlu@gmail.com, \\ alefbakir@gmail.com, erdem.kilavuz@acibadem.com.tr
}

How to cite this paper: Yıldırım, D., Şanlı, D.E.T., Aliş, D., Şahin, M., Bakır, A. and Kilavuz, A.E. (2020) Hashimoto Thyroiditis: Simple Diagnosis with 2D-Shear Wave Elastography. International Journal of Clinical Medicine, 11, 23-31.

https://doi.org/10.4236/ijcm.2020.112003

Received: December 19, 2019

Accepted: February 9, 2020

Published: February 12, 2020

Copyright $\odot 2020$ by author(s) and Scientific Research Publishing Inc. This work is licensed under the Creative Commons Attribution International License (CC BY 4.0).

http://creativecommons.org/licenses/by/4.0/ (c) (i) Open Access

\begin{abstract}
Background: Hashimoto thyroiditis diagnosis is primarily established on clinical and laboratory findings; however, some hashimoto thyroiditis cases are euthyroid and seronegative. Moreover, these patients might also have normal conventional ultrasound findings. Aims: In our study, we aimed to distinguish the typical background characteristics of hashimoto thyroiditis using virtual touch tissue imaging quantification maps obtained with acoustic radiation force impulse imaging. Methods: Our study consisted of 28 hashimoto thyroiditis patients without characteristics of ultrasound findings and 28 healthy subjects. The thyroid parenchymal tissue mechanical properties were analyzed with the virtual touch tissue imaging quantification after ultrasound examination, and then related colored maps were obtained. Shear wave velocities were recorded $(\mathrm{m} / \mathrm{s})$ from the homogenous area where hardest and softest points were closest to each other on virtual touch tissue imaging quantification maps. The difference between the minimum and maximum shear wave velocities for each case $(\Delta v)$ was calculated and recorded. Results: Assessment of virtual touch tissue imaging quantification maps revealed a significant difference between hashimoto thyroiditis and control groups in terms of maximum SWV's, but no significant difference was observed between the minimum shear wave velocities $(\mathrm{p}<0.05)$. Notably, a significant difference was observed when only $\Delta \mathrm{v}$ values were taken into account. In summary, the effects of chronic autoimmune thyroiditis can be dis-
\end{abstract}


tinguished by simply using $\Delta \mathrm{v}$ on virtual touch tissue imaging quantification maps. When the cut-off value of $\Delta \mathrm{v}$ was accepted 0.42 , the diagnosis of hashimoto thyroiditis could be made with $88 \%$ accuracy. Conclusion: We suggest that shear wave velocities measurement on virtual touch tissue imaging quantification maps is a promising method in equivocal hashimoto thyroiditis cases, in which the diagnosis of hashimoto thyroiditis is unachievable with clinical, laboratory and conventional ultrasound findings.

\section{Keywords}

ARFI, Hashimoto Thyroiditis, SWE, SWV, VTIQ

\section{Introduction}

Hashimoto's thyroiditis (HT) is an autoimmune disease that eventually leads to thyroid gland dysfunction. HT has a relatively high prevalence, and predominantly affects women [1] [2] [3]. HT diagnosis established on clinical and laboratory findings; however, $5 \%$ and $13 \%$ of all $\mathrm{HT}$ cases are euthyroid and seronegative respectively while $10 \%$ of the female population have positive anti-TPO and Anti-Tg serology without HT. Furthermore, thyroidectomy specimens of some cases, whose clinical and laboratory findings are incompatible with HT, were diagnosed as HT after the histopathological analysis [4] [5] [6]. These findings suggested that the laboratory and clinical findings alone might not be enough for the diagnosis in some cases. However, despite inconsistencies of the clinical and laboratory aspects aforementioned above, the clinical findings and blood biochemical analyses generally were considered as adequate for the diagnosis of the disease, and additional thyroid ultrasound (US) examination was not commonly requested for the HT diagnosis. On the other hand, HT cases are mostly followed up with the periodic US owing to the elevated risk of papillary thyroid neoplasms. Nevertheless, despite not routinely requested, US examination might be a problem-solving tool in some patients with equivocal clinical and laboratory findings. However, some of the euthyroid and seronegative HT patients might not have noticeable characteristic parenchymal changes of HT disease on US. Moreover, conventional US findings are qualitative, subjective and operator dependent.

Recent studies claimed that shear wave elastography (SWE), a novel elastographic method that quantifies the elasticity of structures by tracking shear waves passing through them, aids to recognize and discriminate the parenchymal pattern in HT [7] [8].

In this study, we aimed to test whether it is possible to recognize background parenchymal changes occurring in thyroid gland in equivocal HT, whose grayscale findings are not characteristic for HT, using virtual touch tissue imaging quantification (VTIQ) maps obtained with acoustic radiation force impulse imaging. 


\section{Materials and Methods}

This prospective study was conducted between January 2014 and January 2015. The study was approved by the local ethics committee, and written consent was obtained from all the patients. The patients with a normal blood level of thyrotropin receptor antibodies (TRAbss $<9.0 \mathrm{U} / \mathrm{L}$ ), high levels of anti-TPO (thyroid peroxidase antibodies, $0-60 \mathrm{IU} / \mathrm{ml}$ ) and anti-Tg (thyroglobulin antibodies, 0 $60 \mathrm{IU} / \mathrm{ml}$ ) were exclusively selected for the patient group. All patients had to have normal thyroid function tests (TSH: $0.51-4.94 \mathrm{uIU} / \mathrm{ml}$, fT4: $10.7-18.4$ pmol/l, fT3: $3.93-7.70 \mathrm{pmol} / \mathrm{l}$ ) to be included in the study since our aim was to evaluate HT patients with obscure clinical findings. Therefore, diagnosis of HT relied on thyroid autoantibodies and clinical findings. All patients were questioned and investigated particularly to exclude any other disease or condition, which might elevate anti-TPO and anti-Tg autoantibodies to ensure elevated levels of autoantibodies were secondary to HT rather than any other systemic or local disease. A single radiologist evaluated all the patients (D.Y with 20 years of ultrasound and 9 years of elastography experience) using Siemens S2000 Helix (US-Siemens Medical Solutions, Erlangen, Germany) with 9-Hz linear transducer. All examinations were performed from the right lobe of the thyroid gland in the axial plane. A detailed grayscale US examination was performed before SWE measurement. All of the patients had to have normal thyroid volumes but not to have parenchymal changes specific to HT disease, prominent heterogeneity and pseudonodular appearance, which might substantially suggest HT [9] [10] [11]. Patients with abnormal thyroid volume and parenchymal changes on US were excluded from the study. Patients were asked to hold their breath for 5 seconds after normal inspiration for SWV imaging. The operator placed transducer providing gentle skin contact without applying compression. VTIQ color maps were obtained in which the blue color shows soft, and the red color shows hard tissue. On VTIQ maps, shear wave velocity (SWV) was recorded $(\mathrm{m} / \mathrm{s})$ from a homogenous area where the hardest and softest points were closest to each other at the same depth. The observer captured five separate VTIQ color maps, and then chose the most appropriate one to perform SWV measurements. The maximum SWV value, measured from the hardest point, and minimum SWV value, measured from the softest point, and the difference between the minimum and maximum speed rate for each case $(\Delta v)$ were calculated. Figure 1 demonstrates SWV measurements on a SWE colored map.

\section{Statistical Analyses}

Independent Student t-Test or Mann Whitney U Test was used for testing the difference between groups for independent samples depending on normal distributed. The normal distribution of the data was tested by Shapiro-Wilk test. Receiver operating characteristic (ROC) curve analysis was carried out to calculate $95 \%$ confidence intervals of the area under the curve (AUC) to identify the best cut off points of difference between the minimum and maximum SWV 


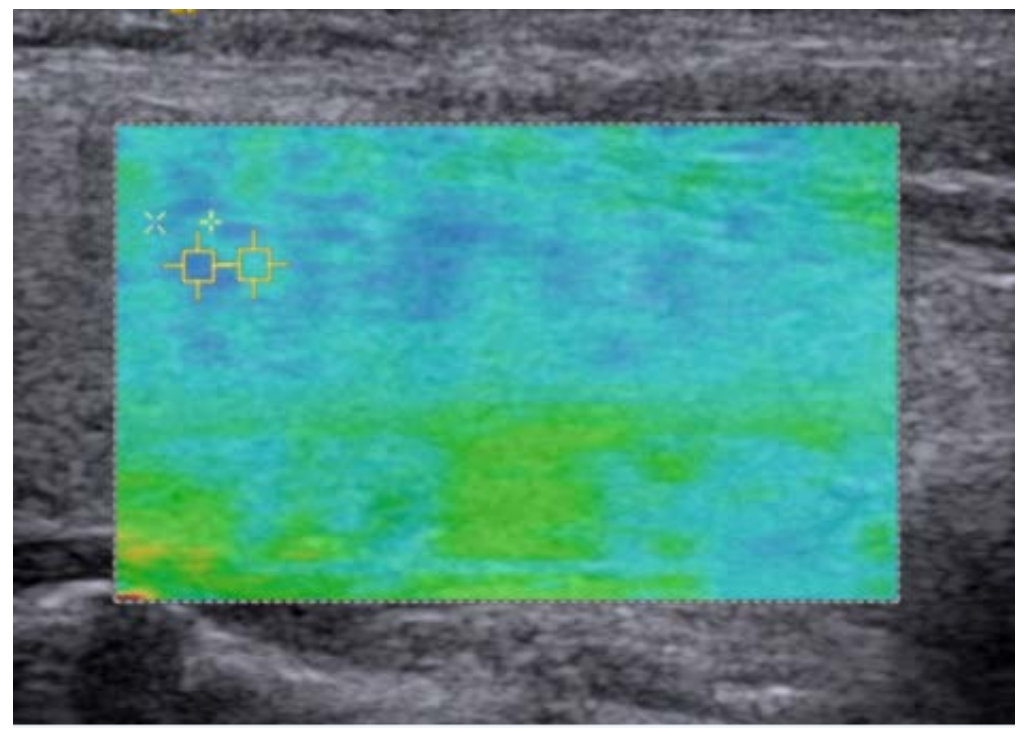

Figure 1. A 35-year-old female patient with HT. The SWV measurement is performed from the homogenous area where hardest and softest points were closest to each other at the same depth. $\mathrm{x}$ : softest point, $1.79 \mathrm{~m} / \mathrm{s}$; +: hardest point, $2.25 \mathrm{~m} / \mathrm{s} . \Delta \mathrm{v}$ is calculated as 0.46 , which is higher than the cut-off value $(0.42 \mathrm{~m} / \mathrm{s})$, suggesting $\mathrm{HT}$.

$(\Delta v)$. The highest value for Youden's index was accepted as the best cutoff value. Youden's index was obtained from coordinates of the curve and calculated by " $J$ $=\max [\mathrm{SN}+\mathrm{SP}]-1$ ". The sensitivity and specificity of the cutoff values were also presented. All data analysis was performed with SPSS 19 for windows and was reported with $95 \%$ confidence intervals, $p<0.05$ was considered significant.

\section{Results}

Finally, 28 patients (27 female, 1 male, mean age: 37 ), and 28 healthy participants (27 female, 1 male, mean age: 39 ) were included in the study. The mean age of the patients was $33.4 \pm 5.2$ years and the mean age of the healthy controls was $32.3 \pm 4.8$ years. There was no significant difference in demographic characteristics between the study and control groups. There was a significant difference the mean maximum SWV values of the patients (was $2.36 \pm 0.22 \mathrm{~m} / \mathrm{s}$ ), and the control group $(2.08 \pm 0.34 \mathrm{~m} / \mathrm{s})(P=0.001)$. The mean minimum velocity values were not statically differs between the patient $(1.83 \pm 0.20 \mathrm{~m} / \mathrm{s})$ and the control group $(1.84 \pm 0.30 \mathrm{~m} / \mathrm{s})(P=0.884>0.05)($ Table 1$)$.

Moreover, when $\Delta \mathrm{v}$ value is taken into account, there is a high significance difference among the patients $(0.53 \pm 0.14)$ and the control groups $(0.24 \pm 0.12)$ $(P<0.001)$ (Table 2).

A receiver operative characteristic (ROC) analysis was applied for the cut-off value of the velocity difference, which is a distinctive feature of the study and control groups. Area Under the Curve (AUC) value was remarkably high $(P<$ $0.001, \mathrm{AUC}=0.931,95 \% \mathrm{CI}: 0.87-0.99)$. Accordingly, when the cut-off value as set to 0.42 , the parenchymal ground influenced by HT could be detected with $79 \%$ sensitivity, $96 \%$ specificity, and $88 \%$ accuracy rate (Table 3, Figure 2). 


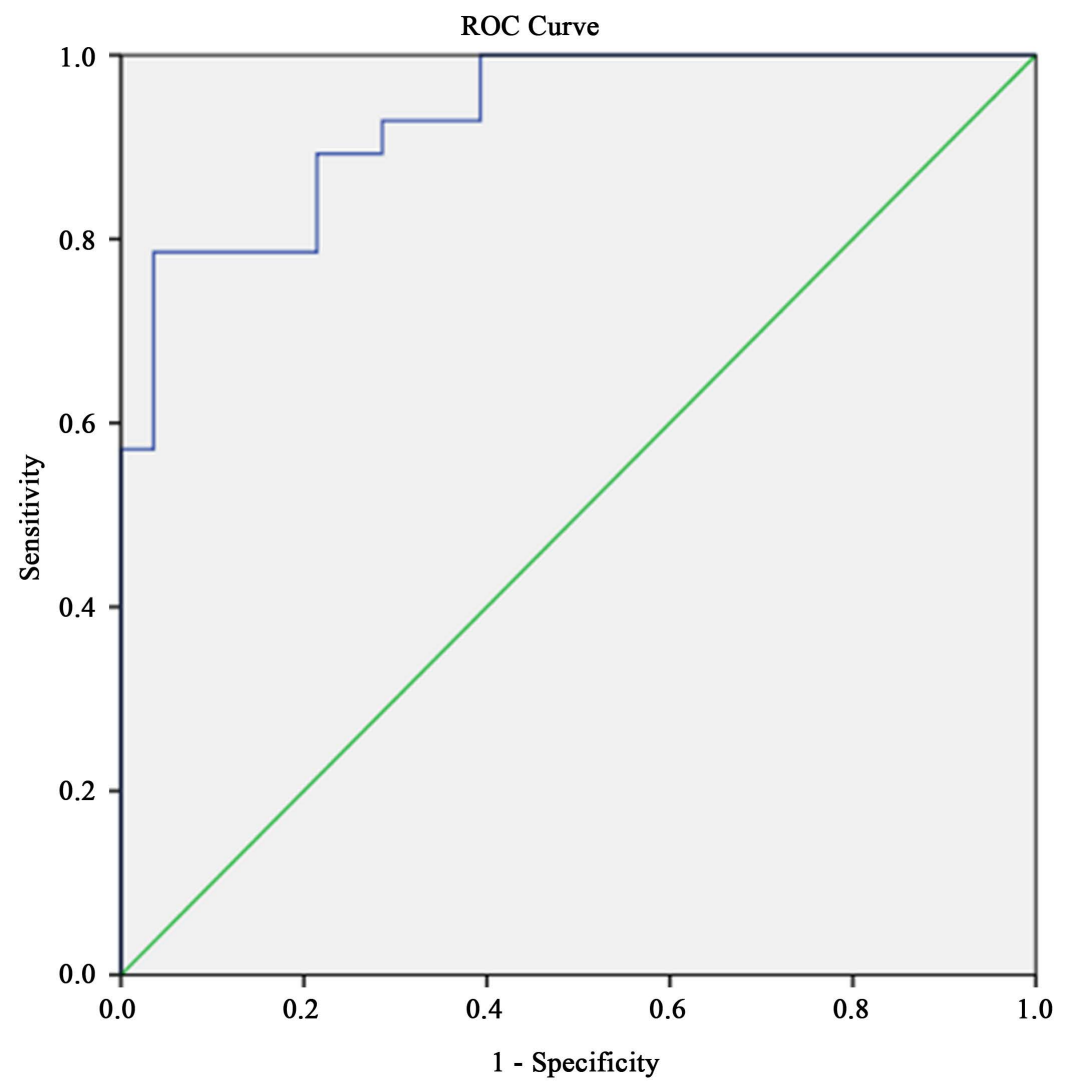

Figure 2. The receiver operative characteristic (ROC) analysis, 79\% sensitivity, 96\% specificity, and $88 \%$ accuracy rate.

Table 1. SWV max and min values in the study and the control groups.

\begin{tabular}{ccccccc}
\hline & & $\mathbf{n}$ & Mean \pm S.D. & Min.-Max. & Median & p \\
\hline \multirow{2}{*}{ Max } & Control & 28 & $2.08 \pm 0.34$ & $1.42-2.66$ & 2.02 & \\
& Study & 28 & $2.36 \pm 0.22$ & $1.90-2.88$ & 2.34 & $0.001^{\mathrm{a}^{*}}$ \\
& Control & 28 & $1.84 \pm 0.30$ & $1.22-2.35$ & 1.88 & $0.884^{\mathrm{a}}$ \\
\hline
\end{tabular}

a.Independent samples test, ${ }^{\star}$ significant.

Table 2. SWV difference in the study and the control groups.

\begin{tabular}{ccccccc}
\hline & & $\mathrm{n}$ & Mean \pm S.D. & Min. - Max. & Median & $\mathrm{p}$ \\
\hline $\begin{array}{c}\text { SWV difference } \\
(\Delta \mathbf{v})\end{array}$ & Control & 28 & $0.24 \pm 0.12$ & $0.04-0.49$ & 0.21 & \\
& Study & 28 & $0.53 \pm 0.14$ & $0.26-0.74$ & 0.55 & \\
\hline
\end{tabular}

a.Independent samples test, ${ }^{*}$ significant.

Table 3. ROC analysis with related Figure 2.

\begin{tabular}{ccccccc}
\hline & Cut-off & SN. (\%) & SP. (\%) & Accuracy (\%) & AUC (\%) & p \\
\hline SWV difference $(\Delta \mathrm{v})$ & 0.42 & $79 \%$ & $96 \%$ & $88 \%$ & 0.931 & $<0.001^{\mathrm{a}^{*}}$ \\
\hline
\end{tabular}

a.ROC curve, ${ }^{*}$ significant. 


\section{Discussion}

Grayscale ultrasound findings like heterogeneous echo-structure with pseudo nodules, and Doppler US findings such as increased or hierarchical vascularity are distinct features for HT; nonetheless, these modalities have low diagnostic value owing to their subjective characteristics, and also their various presenting degrees in different cases. Even though several ultrasound texture analysis studies aimed to overcome these problems, since the post-processing is relatively sophisticated and nonspecific, all suggested techniques gained no acceptance for routine daily ultrasonography practice. Furthermore, some studies pointed out that texture analyses have no superiority over conventional grayscale US assessment in the differentiation of HT [12] [13].

SWE is mainly used to differentiate benign and malignant thyroid nodules, but it could also be used in the diagnosis of HT. There are a few numbers of studies available about SWE in the literature, which primarily aimed to differentiate benign and malignant nodules in HT patients [14] [15] [16] [17]. Some other SWE studies sought to discover the probable background elasticity changes developed by chronic autoimmune thyroiditis [14] [18] [19] [20].

The SWV values that are acquired with different hardware and/or software may affect the results. Fukuhara et al. and Hekimoglu et al. conducted two separate studies, and concluded that thyroid glands of the cases with chronic autoimmune thyroiditis (CAT) have relatively higher SWV values. They observed SWV values that reach up to $2.56 \pm 0.57 \mathrm{~m} / \mathrm{s}$ and $2.56 \pm 0.30 \mathrm{~m} / \mathrm{s}$ in the patients with CAT [19] [20]. On the other hand, Magri's study in which the measurement obtained and recorded in kilopascal unit suggested that there was no statistically significant difference between parenchymal ground SWV values of normal and HT cases [14].

Senile or sequela (post-treatment) changes may also have a heterogeneous hypo-echogenic parenchymal pattern; thus, mimic the appearance of HT background. More information could be gathered about the disease when these parenchymal patterns are correctly assessed and differentiated from the HT [21].

There are some reported cases with postoperative histopathological definite HT diagnosis, in which clinical and laboratory data were not compatible with HT [4] [5] [6]. In light of all the aforementioned issues, it is vital to diagnose HT at an early stage, especially in the areas where the disease is endemic.

Our study differs from the others by investigating $\Delta \mathrm{v}$ value, which we suggest that it could be accounted as a more reliable method to detect parenchymal background changes in HT. We highlight that both normal patients' thyroid parenchyma and HT patients' thyroid parenchyma might demonstrate soft and hard areas on VTIQ color map, which might arise some challenges to the operators, especially inexperienced ones, in deciding to choose the hardest or softest area on VTIQ map. Unfortunately, inappropriate detection of the hardest or softest area might yields improper measurements might yield false negative or positive results on SWE examination. On the other hand, given the heterogeneity of the gland in HT disease, SWV differences within the gland are substantially 
more prominent than the normal gland. Thus, if even the operator does not visually able to determine softest or hardest area, calculating the difference, namely $\Delta \mathrm{v}$ value, the value between the softest and hardest area that visually and subjectively identified by the observer, might be more accurate owing to prominent stiffness differences within gland due to parenchymal background changes of HT.

Nevertheless, we acknowledge that there are several limitations in our study. First, our study is the absence of Graves' disease, which is the most important group of diseases that can mimic HT. Hence, our method should be tested on larger series in the centers with frequent cases of Graves' and HT. Second, we did not be able to do histological confirmation, but we had no chance but to rely on the diagnosis we achieved with clinical-laboratory thyroid function indicators and autoantibodies since thyroid biopsy or surgery is not necessarily indicated to diagnose HT in our country. Third, we had a small number of participants since we strictly formed our inclusion criteria as we mentioned in the materials and methods section. Fourth, we did not test the reproducibility of the SWV measurements; however, many studies have tested and demonstrated both inter and intraobserver correlation of the SWV measurements of the thyroid gland is considerably high with relatively straightforward learning curve [22]. In this study, our cases were evaluated without separation of HT into acute exacerbation period or subacute-chronic period. SWV and $\Delta \mathrm{v}$ values may be measured in larger patient populations divided into these groups according to clinical, laboratory and sonographic characteristics. Finally, we did not evaluate the relationship between the SWV values and the laboratory values of the patients since the main scope of our study was to simply offer an auxiliary method in the diagnosis of HT. The correlation between the SWV measurements and the laboratory results of the patients should be assessed in the future studies with a larger population.

\section{Conclusion}

In conclusion, with the contribution of SWE, the velocity difference $(\Delta v)$ between the hardest and the softest areas on thyroid gland parenchyma could be determined through SWV colored maps (VTIQ); thus, it would be straightforward to define whether the changes on the ground are secondary to HT or not. When the cut off value for $\Delta v$ is taken as $0.42(\mathrm{~m} / \mathrm{s})$, background influenced by HT can be detected with $96 \%$ sensitivity and $88 \%$ accuracy by this method alone. Even the other parameters are not available to utilize or impractical to use, $\Delta \mathrm{v}$ may provide valuable information in the one-stop diagnosis of the HT. However, further studies that would test our findings in larger series with other diffuse parenchymal diseases, especially Graves' disease, are needed to reveal the actual value and availability of this method.

\section{Conflicts of Interest}

All authors declare no conflict of interest. 


\section{Ethical Statements}

All procedures followed were in accordance with the ethical standards of the responsible committee on human experimentation (institutional and national) and with the Helsinki Declaration of 1964 and later versions.

Informed consent was obtained from all patients for being included in the study.

\section{Financial Disclosure}

The authors have no relevant financial interest in this article.

This original article has been presented as e-poster in ECR 2017.

English of this manuscript was checked by a language editing company.

\section{References}

[1] Ajjan, R.A. and Weetman, A.P. (2015) The Pathogenesis of Hashimoto's Thyroiditis: Further Developments in Our Understanding. Hormone and Metabolic Re search, 47, 702-710. https://doi.org/10.1055/s-0035-1548832

[2] Jaume, J.C. (2007) Endocrine Autoimmunity. In: Greenspan's Basic \& Clinical Endocrinology, Springer, Berlin, 59-79.

[3] Kumar, V. (2010) 24: The Endocrine System. Robbins and Cotran Pathologic Mechanisms of Disease. 8th Edition, Elsevier, Philadelphia, PA, 1111-1205.

[4] Tomer, Y. and Huber, A. (2009) The Etiology of Autoimmune Thyroid Disease: A Story of Genes and Environment. Journal of Autoimmunity, 32, 231-239.

[5] Hiromatsu, Y., Satoh, H. and Amino, N. (2013) Hashimoto's Thyroiditis: History and Future Outlook. Hormones (Athens), 12, 12-18.

https://doi.org/10.1007/BF03401282

[6] Effraimidis, G. and Wiersinga, W.M. (2014) Mechanisms in Endocrinology: Autoimmune Thyroid Disease: Old and New Players. European Journal of Endocrinology, 170, 241-252. https://doi.org/10.1530/EJE-14-0047

[7] Tasli, F., Özkök, G., Argon, A., et al. (2014) The Role of IgG4 (+) Plasma Cells in the Association of Hashimoto's Thyroiditis with Papillary Carcinoma. APMIS, 122, 1259-1265. https://doi.org/10.1111/apm.12297

[8] Noureldine, S.I. and Tufano, R.P. (2015) Association of Hashimoto's Thyroiditis and Thyroid Cancer. Current Opinion in Oncology, 27, 21-25. https://doi.org/10.1097/CCO.0000000000000150

[9] Chaudhary, V. and Shahina, B. (2013) Thyroid Ultrasound. The Indian Journal of Endocrinology and Metabolism, 17, 219-227. https://doi.org/10.4103/2230-8210.109667

[10] Langer, J.E., Khan, A., Nisenbaum, H.L., et al. (2001) Sonographic Appearance of Focal Thyroiditis. American Journal of Roentgenology, 176, 751-754. https://doi.org/10.2214/ajr.176.3.1760751

[11] Yeh, H.C., Futterweit, W. and Gilbert, P. (1996) Micronodulation: Ultrasonographic Sign of Hashimoto Thyroiditis. Journal of Ultrasound in Medicine, 15, 813-819. https://doi.org/10.7863/jum.1996.15.12.813

[12] Kim, G.R., Kim, E.-K., Kim, S.J., et al. (2016) Evaluation of Underlying Lymphocytic Thyroiditis With Histogram Analysis Using Grayscale Ultrasound Images. Journal of Ultrasound in Medicine, 35, 519-526. 
[13] Nam, S.J., Yoo, J., Lee, H.S., et al. (2016) Quantitative Evaluation for Differentiating Malignant and Benign Thyroid Nodules Using Histogram Analysis of Grayscale Sonograms. Journal of Ultrasound in Medicine, 35, 775-782.

[14] Magri, F., Chytiris, S., Capelli, V., et al. (2012) Shear Wave Elastography in the Diagnosis of Thyroid Nodules: Feasibility in the Case of Coexistent Chronic Autoimmune Hashimoto's Thyroiditis. Clinical Endocrinology, 76, 137-141. https://doi.org/10.1111/j.1365-2265.2011.04170.x

[15] Han, R., Li, F., Wang, Y., Ying, Z. and Zhang, Y. (2015) Virtual Touch Tissue Quantification (VTQ) in the Diagnosis of Thyroid Nodules with Coexistent Chronic Autoimmune Hashimoto's Thyroiditis: A Preliminary Study. European Endocrinology, 84, 327-331. https://doi.org/10.1016/j.ejrad.2014.11.005

[16] Liu, B., Liang, J., Zhou, L., et al. (2015) Shear Wave Elastography in the Diagnosis of Thyroid Nodules with Coexistent Chronic Autoimmune Hashimoto's Thyroiditis. Otolaryngology_Head and Neck Surgery, 153, 779-785. https://doi.org/10.1177/0194599815600149

[17] Liu, B.-J., Xu, H.-X., Zhang, Y.-F., et al. (2015) Acoustic Radiation Force Impulse Elastography for Differentiation of Benign and Malignant Thyroid Nodules with Concurrent Hashimoto's Thyroiditis. Medical Oncology, 32, 1-9.

[18] Bhatia, K.S., Tong, C.S., Cho, C.C., et al. (2012) Shear Wave Elastography of Thyroid Nodules in Routine Clinical Practice: Preliminary Observations and Utility for Detecting Malignancy. European Radiology, 22, 2397-2406.

[19] Fukuhara, T., Matsuda, E., Izawa, S., Fujiwara, K. and Kitano, H. (2015) Utility of Shear Wave Elastography for Diagnosing Chronic Autoimmune Thyroiditis. Journal of Thyroid Research, 2015, Article ID: 164548. https://doi.org/10.1155/2015/164548

[20] Hekimoglu, K., Donmez, F.Y., Arslan, S., et al. (2015) The Role of Shear Wave Elastography in the Diagnosis of Chronic Autoimmune Thyroiditis. Medical Ultrasonography, 17, 322. https://doi.org/10.11152/mu.2013.2066.173.khu

[21] Shin, D.Y., Kim, E.-K. and Lee, E.J. (2010) Role of Ultrasonography in Outcome Prediction in Subclinical Hypothyroid Patients Treated with Levothyroxine. Endocrine Journal, 57, 15-22. https://doi.org/10.1507/endocrj.K09E-154

[22] Dillman, J.R., Chen, S., Davenport, M.S., et al. (2015) Superficial Ultrasound Shear Wave Speed Measurements in Soft and Hard Elasticity Phantoms: Repeatability and Reproducibility Using Two Ultrasound Systems. Pediatric Radiology, 45, 376-385. 